\title{
Educación jurídica y reproducción social en Colombia ${ }^{*}$
}

\author{
Legal Education and Social Reproduction in Colombia \\ Educação jurídica e reprodução social na Colômbia
}

María Adelaida Ceballos Bedoya **

FeCHA DE RECEPCIÓN: 30 DE ENERO DE 2017. FeCHA DE ACEPTACIÓN: 15 DE JUNIO DE 2017

DOI: http://dx.doi.org/10.12804/revistas.urosario.edu.co/sociojuridicos/a.5373

Para citar este artículo: Ceballos Bedoya, M. A. (2018). Educación jurídica y reproducción social en Colombia. Estudios Socio-Jurídicos, 20(1), 77-105. doi: http://dx.doi.org/10.12804/revistas.urosario.edu.co/sociojuridicos/a.5373

\section{RESUMEN}

El propósito de este artículo es estudiar las relaciones que existen entre la calidad de los programas de Derecho en Colombia y el nivel socioeconómico de sus estudiantes. El texto plantea dos conclusiones: primero, la oferta de educación jurídica en Colombia es amplia y heterogénea, y se caracteriza por un predominio de los programas privados de bajo costo y baja calidad. Segundo, el ingreso a los programas de alta calidad depende del origen social de los estudiantes, pues a ellos solo acceden quienes tienen alta capacidad de pago (en el sector privado) o resultados académicos sobresalientes (en el sector público). Esto sugiere que la educación jurídica, en lugar de reducir las desigualdades sociales, contribuye a reproducirlas.

Palabras clave: educación jurídica, sociología de la educación, desigualdad, educación superior, abogados.

* Este artículo se desprende de la tesis presentada por la autora en la Maestría en Sociología de la Universidad Nacional de Colombia (Bogotá).

** Abogada de la Universidad Eafit (Medellín) y Magíster en Sociología de la Universidad Nacional de Colombia (Bogotá). Investigadora del Centro de Estudios de Derecho, Justicia y Sociedad (Dejusticia) (Bogotá). Correo electrónico: mayaceballos@gmail.com. Orcid://orcid.org/00000002-6139-4061 


\section{ABSTRACT}

The purpose of this article is to study the relationship between the quality of the legal education in Colombia and the socioeconomic status of the law students. This article presents two conclusions. First, the supply of legal education is wide and heterogeneous in Colombia, and it is characterized by the prevalence of low-cost and low-quality private programs. Second, admission to high-quality legal education depends on students' social origin. Only those who have either high economic capacity (in the private sector) or outstanding academic results (in the public sector) are able to access this kind of programs. It suggests that legal education reproduces inequalities instead of reducing them.

Key words: Legal education, sociology of education, inequality, higher education, lawyers.

\section{RESUMO}

O propósito deste artigo é estudar as relações que existem entre a qualidade dos programas de direito na Colômbia e o nível socioeconómico de seus estudantes. $\mathrm{O}$ artigo apresenta duas conclusões: primeiro, a oferta de educação jurídica na Colômbia é ampla e heterogênea, e caracteriza-se por um predomínio dos programas privados de baixo custo e baixa qualidade. Segundo, o ingresso aos programas de alta qualidade depende da origem social dos estudantes, pois a eles só tem acesso quem têm alto poder aquisitivo (no instituições privadas) ou resultados acadêmicos sobressalentes (no instituições públicas). Isto sugere que a educação jurídica, em vez de reduzir as desigualdades sociais, contribui para aumentá-las.

Palavras -chave: educação jurídica, sociologia da educação, desigualdade, educação superior, advogados. 


\section{Introducción}

Los abogados en Colombia tienen una especial relevancia política y social. Cuando son litigantes, tienen en sus manos los bienes, la libertad y la vida de sus representados. Cuando son jueces, tienen la responsabilidad de garantizar el acceso a la justicia de los usuarios, e, incluso, de encargarse de tareas tan arduas como el desmantelamiento del crimen organizado o la interpretación última de la Constitución. Además, las burocracias estatales suelen estar en manos de profesionales del Derecho, de manera que la eficiencia y la transparencia del Estado están estrechamente atadas al talante de los abogados que lo componen.

A pesar de su importancia, las reflexiones académicas sobre los abogados en Colombia son escasas y generalmente tienen poco asidero empírico. Esto se cumple particularmente en el caso de las investigaciones sobre educación jurídica, entendida como la educación formal ofrecida en el nivel de pregrado a los futuros abogados. Así, apenas unas pocas investigaciones se han preocupado por la expansión de la oferta de educación jurídica de baja calidad, y solo algunas de ellas ofrecen datos sobre la evolución cuantitativa de la oferta y la demanda de los pregrados en Derecho (Arbeláez Herrera y Espinosa Pérez, 2002; Espinosa Blanco, 2012; Fuentes Hernández, 2005; García Villegas, 2010; Silva García, 2001). Sin embargo, esas investigaciones no definen qué entienden por programas de calidad ni se ocupan de caracterizar esos programas (y sus estudiantes) desde un punto de vista socioeconómico.

Este artículo busca contribuir a llenar ese vacío mediante la recolección de información oficial sobre la educación jurídica en Colombia. El objetivo es analizar las relaciones que actualmente existen entre la calidad de los pregrados de Derecho y el nivel socioeconómico de sus estudiantes. Las conclusiones a las que llega este artículo son dos: primero, que la oferta de educación jurídica es amplia y heterogénea, y se caracteriza por un predominio de los programas privados de bajo costo y baja calidad. Y, segundo, que esa heterogeneidad está sesgada en términos de clase, pues la oportunidad de acceder a los programas de mayor calidad se limita a los pocos estudiantes que tienen alta capacidad económica (en el caso de los pregrados privados) o que logran obtener resultados académicos sobresalientes (en el sector público). 
Así pues, la oferta de educación jurídica parece estar segmentada en varios niveles o tipos de pregrados, que difieren según su calidad y su costo. Hay un grupo grande de pregrados de bajo costo y baja calidad (especialmente del sector privado), en el cual se forma la mayoría de abogados del país. Los programas de alta calidad, en cambio, son muy pocos y educan a una porción menor de los abogados. Pero la oferta de educación jurídica de alta calidad no solamente es muy pequeña, sino que la posibilidad de ingresar a ella es muy limitada: en el sector oficial, las matrículas no son costosas, pero las universidades imponen filtros académicos de admisión que muy pocos superan. En el sector privado, los filtros académicos son débiles pero las matrículas son muy costosas, de manera que allí pueden ingresar fácilmente los estudiantes que tengan el dinero suficiente para pagarlas, que no son muchos.

Lo anterior implica, por un lado, que predominan los estudiantes y los abogados formados en programas de baja calidad, mientras que los abogados de mayor calidad escasean. Por otro lado, significa que los estudiantes con alta capacidad económica tienen amplísimas oportunidades de acceder a una buena formación en Derecho, mientras que en el caso de los estudiantes con baja capacidad económica (que son la mayoría) esas oportunidades se reducen dramáticamente. Esta situación, vale decir, no es exclusiva del sistema universitario, sino que es la continuación o el reflejo de las desigualdades sociales de la educación básica y media en Colombia, donde el éxito académico está fuertemente atado al origen social de los estudiantes.

Estos hallazgos son problemáticos al menos por dos razones. En primer lugar, porque evidencian que la educación jurídica en Colombia no está contribuyendo a combatir las desigualdades sociales. Bien al contrario, y en la línea de lo que sostiene la teoría de la reproducción social (Bourdieu y Passeron, 1996, 2009), parece que la educación jurídica colombiana reproduce las desigualdades socioeconómicas de los estudiantes, pues les brinda mejores oportunidades educativas a quienes gozan de una mayor herencia cultural y económica. ${ }^{1}$ Esto defrauda las expectativas de muchos de los sectores menos privilegiados de la población, que siguen concibiendo a la educación como una estrategia efectiva de movilidad 
social (Quintero, 2013, p. 10). Además, supone un incumplimiento de las promesas del gobierno nacional, y del Estado social de derecho en general, en torno a la necesidad de nivelar las condiciones de origen de los ciudadanos a través de la educación (DNP, 2014).

En segundo lugar, estos hallazgos serían de por sí preocupantes en relación con cualquier pregrado. Pero en el caso de los programas de Derecho es una situación que puede tener consecuencias sociopolíticas y jurídicas particularmente graves, ya que, por su especial relevancia social, el predominio de los abogados de baja calidad probablemente se traducirá en un desmedro de la administración pública, la justicia y el sistema político. El deber estatal de preocuparse por la cantidad y la calidad de los abogados parece entonces ser mayor que en el caso de otras profesiones que tienen, digamos, un menor impacto social (García Villegas, 2010, p. 23).

El texto se dividirá en cuatro partes. En la primera, se explicará cuál fue la metodología empleada en la elaboración de este artículo. En la segunda, se caracterizarán la oferta y la demanda de educación jurídica en Colombia, tanto en términos cuantitativos como cualitativos, y se mostrará cómo esa oferta está segmentada en varios niveles o tipos de pregrados. En la tercera, se explicará cuáles son las particularidades de acceso y costo de los mejores pregrados en Derecho, para entonces plantear cómo esas particularidades contribuyen a reproducir las desigualdades socioeconómicas de los estudiantes. Finalmente, se propondrán algunas conclusiones.

\section{Metodología}

Para los propósitos de este artículo, se recolectó información oficial y se implementaron técnicas básicas de estadística descriptiva, de una parte, sobre la calidad de los 178 pregrados de Derecho presenciales que se ofrecían en Colombia en 2015; y, de otra parte, sobre el perfil socioeconómico de los estudiantes de dichos pregrados.

En relación con la calidad, se estudiaron dos indicadores. Primero, las acreditaciones de alta calidad de los pregrados de Derecho y de las instituciones de educación superior (IES) que los ofrecían en el año 2015. 
Así, para poder operar legalmente, todos los pregrados deben contar con un registro calificado, pero voluntariamente pueden someterse a un proceso de acreditación de alta calidad. Este proceso es conducido por el Consejo Nacional de Acreditación, pero el reconocimiento final de acreditación es emitido por el Ministerio de Educación Nacional (MEN). También las IES tienen la opción de someterse a estos procesos. Puede haber programas acreditados en IES no acreditadas y viceversa.

El segundo indicador de calidad fue el promedio de los puntajes obtenidos por los estudiantes de cada pregrado de Derecho en las pruebas Saber Pro (años 2005 a 2015). ${ }^{2}$ Las pruebas Saber Pro son los exámenes de Estado aplicados por el Icfes a los estudiantes universitarios al finalizar sus cursos de pregrado. Desde 2009 son un requisito obligatorio para obtener el título profesional. En este caso, 40 programas ( 25 privados y 15 oficiales) fueron excluidos del análisis, pues, a causa de su reciente creación, no contaban todavía con reportes de resultados en estas pruebas.

Estos dos criterios de calidad han sido, ciertamente, objeto de muchas críticas y revisiones. Sin embargo, constituyen indicadores objetivos que provienen de fuentes independientes y que, al ser evaluados conjuntamente, pueden cuando menos mostrar algunas tendencias gruesas del estado actual de la calidad de la educación jurídica. ${ }^{3}$

En cuanto al perfil socioeconómico de los estudiantes, se indagó también por dos indicadores. Por una parte, se recolectó información sobre el costo aproximado de matrícula de cada pregrado en 2015. ${ }^{4}$ A partir de allí, se construyeron cinco escalas de costo, desde 'baja' a 'muy alta'. Para construir esas escalas, primero se obtuvo la media del costo de las matrículas privadas (que fue de 3388000 pesos) y la desviación estándar de los datos (\$1722640). Los valores entre la media del costo y una desviación estándar hacia arriba se clasificaron como 'medio altos';

2 La información sobre las acreditaciones se obtuvo del sistema SNIES del Ministerio de Educación Nacional (MEN). En lo relativo a las pruebas Saber Pro, la información del período 20052010 vino del portal del Icfes Interactivo - Reportes históricos, mientras que la del período 2012-2015 vino del sistema FTP del Icfes.

3 Algunas críticas a estos criterios de calidad pueden encontrarse en Gómez Campo (2014) y en el Observatorio de la Universidad Colombiana (2015).

4 La información de los costos de matrícula de los programas provino del SNIES, del Observatorio de la Universidad Colombiana, de las páginas web de los respectivos programas y de respuestas a derechos de petición. 
los valores que quedaron entre una y dos desviaciones estándar hacia arriba, como 'altos'; y a partir de dos desviaciones estándar, como 'muy altos'. Los valores entre la media y una desviación estándar hacia abajo se clasificaron como 'medio bajos'; y entre una y dos desviaciones estándar hacia abajo, como 'bajos'.

Por otra parte, se analizó el estrato socioeconómico promedio del total de estudiantes de cada programa en 2015. ${ }^{5}$ No se dispone de información ni sobre el estrato de cada estudiante ni sobre otras variables socioeconómicas distintas al estrato, lo cual permitiría hacer diagnósticos más precisos en este punto. Sin embargo, el estrato socioeconómico (entendido como una forma aproximativa a la clase social) y el costo de matrícula pueden mostrar unas tendencias generales y agregadas sobre la posible composición socioeconómica del estudiantado de cada programa.

\section{El estado actual de la oferta y de la demanda de educación jurídica}

Esta sección está dedicada a dar cuenta, primero, de la magnitud de la oferta y la demanda de la educación jurídica; segundo, de los problemas de calidad de los pregrados de Derecho; y tercero, de los distintos tipos de pregrados que se ofrecen, según su costo y su calidad.

\subsection{El aumento de la educación jurídica}

La educación jurídica se enmarca en un contexto más amplio de desregulación de la educación superior en Colombia. Según algunos expertos, esa desregulación comienza esencialmente con la expedición de la Ley 30 de 1992, que desarrolló el principio de autonomía universitaria consagrado en la Constitución Política (art. 69), imponiendo un esquema

5 La información sobre los estratos de cada programa se obtuvo del portal SPADIES del Ministerio de Educación Nacional y de respuestas a derechos de petición. Se analizó solamente la información del año 2015 porque era el año en el que había menor subreporte y porque sus promedios no mostraban una variación significativa en relación con los que se reportaban en años anteriores. 
de autonomía institucional que redujo el papel estatal y amplió el margen de acción del mercado (Gómez Campo, 2000, 2014).

En el caso concreto de la carrera de Derecho, después de 1992 el Estado colombiano ha tratado en varias ocasiones de regular la creación de nuevos pregrados y de imponer estándares de calidad más estrictos. ${ }^{6} \mathrm{Sin}$ embargo, esos intentos han sido generalmente precarios e insuficientes, pues no han conseguido frenar el crecimiento de la oferta de pregrados (principalmente privados), y tampoco han podido establecer regulaciones o controles rigurosos en términos de calidad. De hecho, actualmente en Colombia no existe ni siquiera una regulación específica sobre las características de calidad que deben reunir los pregrados de Derecho. ${ }^{7}$

En medio de un déficit de regulación y vigilancia estatales, en las últimas décadas se ha presentado entonces un marcado crecimiento de la oferta de educación jurídica, con un claro protagonismo de la oferta privada. Así, mientras en 1993 había 38 programas, en 2015 la cifra llegó a 178 (Salamanca, 2010a, p. 113) (figura 1). ${ }^{8}$ De estos 178 pregrados de 2015, 142 (es decir, el 80\%) pertenecen al sector privado y solamente 36 pertenecen al oficial. En suma, los pregrados crecieron un 368\% en 22 años, lo cual equivale a más de seis pregrados nuevos por año. ${ }^{9}$

De la mano del crecimiento de la oferta, se ha presentado también una ascendente producción de estudiantes. Entre los semestres 2000-1 y 2015-1, la cantidad de matriculados aumentó más del 100\%, pasando de 55531 a 126264 estudiantes por semestre. Como en el caso de los programas, el crecimiento de la población estudiantil ha estado principalmente jalonado por el sector privado, que pasó de 44629 matriculados en 2000-1 a 102179 en 2015-1. El sector oficial también creció

6 Sobre algunos de estos intentos regulatorios y sobre el rol predominante del sector privado en el crecimiento de la oferta de educación jurídica, ver Salamanca (2010a, 2010b).

7 Anteriormente, los pregrados de Derecho estaban específicamente regulados por la $\mathrm{Re}^{-}$ solución 2768 de 2003 del Ministerio de Educación, que desarrollaba el Decreto 2566 de 2003. No obstante, este decreto fue declarado nulo por el Consejo de Estado (2011) y con ello quedó también derogada la resolución sobre los programas de Derecho. En 2015, el Ministerio de Educación y el Ministerio de Justicia y del Derecho (2016) promovieron una iniciativa para restablecer la regulación específica de los programas jurídicos, pero finalmente no dio frutos.

8 Estas cifras se refieren al número de pregrados ofrecidos por cada institución en cada municipio, sin discriminar por el número de jornadas ofrecidas dentro de esos programas.

9 Según el SNIES, en junio de 2017 la cifra era ya de 190 pregrados en Derecho. 
proporcionalmente, pero tuvo un aumento menos marcado que el sector privado, pasando de 10902 a 24085 matriculados en el mismo período.

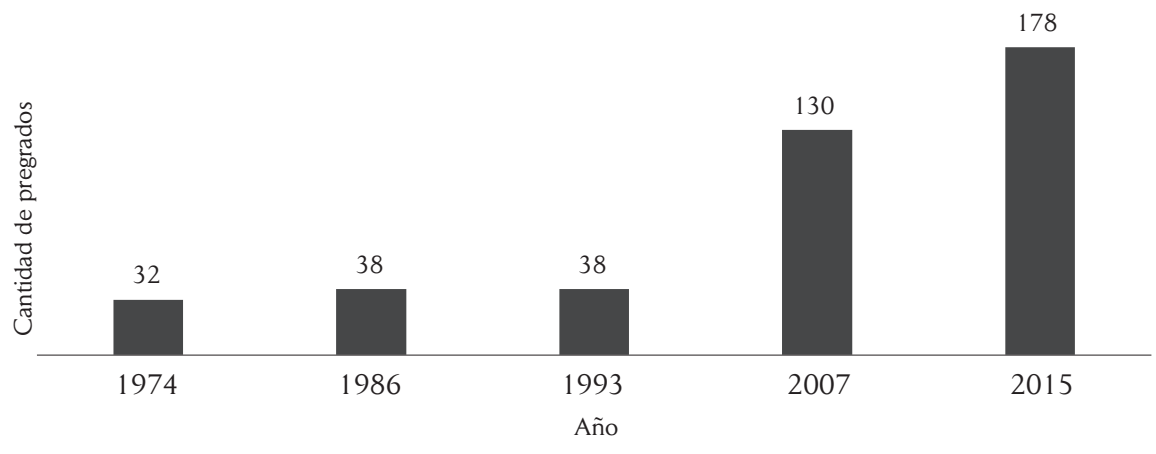

Figura 1. Cantidad de pregrados de Derecho (1974-2015)

Fuente: elaboración de la autora a partir de Salamanca (2010a, p. 114) y Ministerio de Educación Nacional-SNIES (2015).

El crecimiento de los estudiantes se ha traducido también en un aumento de la cantidad de profesionales del Derecho. En 1991, fueron registradas alrededor de 4000 tarjetas profesionales, mientras que en 2014 se registraron más de 17000 (Unidad de Registro Nacional de Abogados, 2009, 2014). La magnitud de estas cifras quizá puede hacerse más clara si se piensa que, según el Observatorio Laboral para la Educación, en 2015 se graduaron 13915 abogados de las facultades de Derecho del país, mientras que se graduaron 4699 médicos, 2354 economistas, 1076 biólogos y solamente 504 sociólogos y 153 geógrafos.

Pero no solo ha crecido la cantidad de abogados en términos absolutos, sino también la proporción de abogados por habitante. En el año 2000 había una tasa de 254 abogados por 100000 habitantes y en 2014 esa tasa era de 496 (DANE, 2005; Pérez Perdomo, 2005; Unidad de Registro Nacional de Abogados, 2014). Más aún, Colombia se destaca por tener una de las tasas de abogados por habitante más altas del mundo: en 2014, la tasa de abogados en Colombia era de 496; en Perú, de 427; en España, de 291; en Alemania, de 200; y en Finlandia, de 47 (European Commission, 2016, figura 33; Unidad de Registro Nacional de Abogados, 2014). 


\subsection{Los problemas de calidad de la educación jurídica}

La abundante oferta de educación jurídica podría no ser un problema si esta fuera de buena calidad. Pero otra cosa ocurre en Colombia, pues, como se muestra a continuación, los programas de alta calidad no solamente escasean, sino que educan a una pequeña porción de los abogados.

Como se anunció en la metodología, se evaluaron dos indicadores de calidad: primero, las acreditaciones de alta calidad; y, segundo, los resultados en las pruebas Saber Pro. El primer indicador de la calidad se refiere entonces a las acreditaciones de alta calidad que el MEN les otorga tanto a los pregrados como a las instituciones que ofrecen esos pregrados. Pues bien, de los 178 pregrados de Derecho vigentes en 2015, solamente 20 ( 5 oficiales y 15 privados) contaban con acreditación de alta calidad y eran ofrecidos por IES acreditadas. Es decir, solamente 20 pregrados (el $11 \%$ del total) gozaban del doble rasero de acreditación de calidad. En 2015, estos pregrados albergaban al 15\% de los estudiantes matriculados: $11 \%$ en el sector privado y $4 \%$ en el oficial.

Paralelamente, los pregrados sin ninguno de los dos raseros de calidad (138 en total) representaban el 78\% de la oferta y recibían al 66\% de los estudiantes. Por su parte, los pregrados que solo tenían acreditación del programa (no de la IES) representan el $8 \%$ de los pregrados y el 18\% de los estudiantes (figura 2).

Estos resultados se refuerzan al analizar el segundo indicador de calidad, que es el puntaje promedio de cada programa en las pruebas Saber Pro, pues se encontró que hay una fuerte relación entre los puntajes en las pruebas Saber Pro y las acreditaciones de alta calidad (especialmente, la acreditación de la IES). Así, los mejores promedios suelen ubicarse en las instituciones acreditadas; y, en particular, en los programas con doble rasero de calidad. Por su parte, los programas sin doble rasero tienden a obtener los promedios más bajos en comparación con los otros tipos de programas, ${ }^{10}$ mientras que los programas acreditados de IES no acreditadas mostraron también una tendencia a los resultados bajos. ${ }^{11}$

10 Estos resultados en parte se confirman en el Ministerio de Educación y Ministerio de Justicia (2016, p. 5).

11 Como se advirtió en la metodología, 40 programas fueron excluidos de este análisis. Ninguno de ellos tenía acreditación de alta calidad y solamente uno era ofrecido por una IES acreditada. 


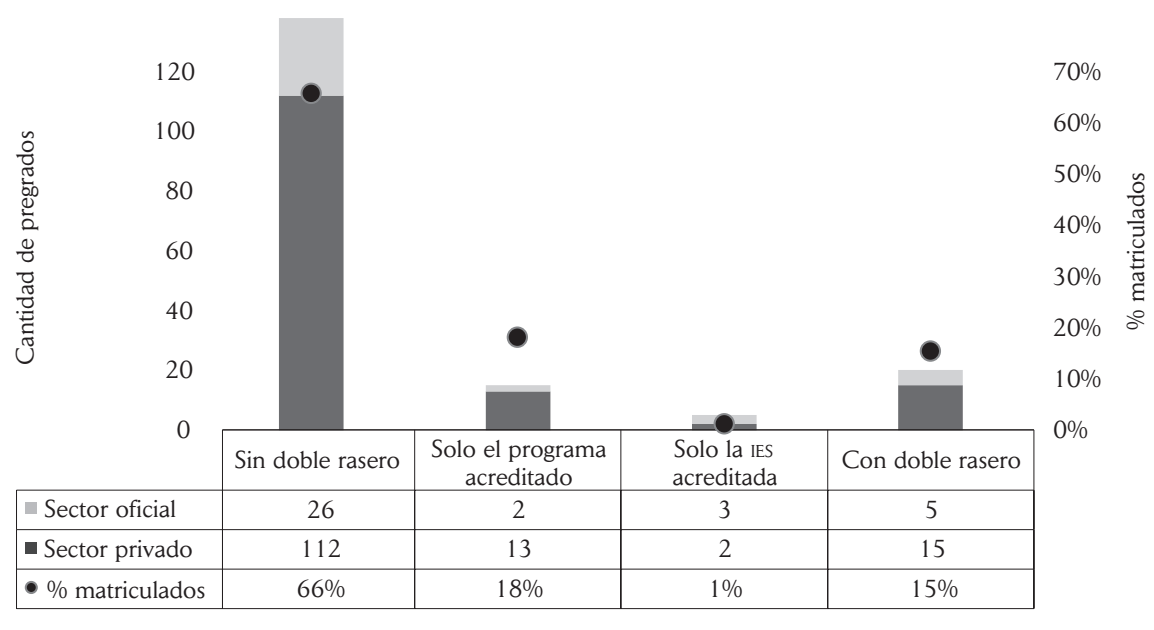

Figura 2. Cantidad de pregrados de Derecho según acreditaciones y porcentaje de matriculados (2015)

Fuente: elaboración de la autora a partir de MEN-SNIES (2015).

Según lo dicho, en la educación jurídica escasean los programas que gozan del doble rasero, que son además los que suelen obtener los resultados más destacados en las pruebas estatales. Pero esos programas no solo son minoría, sino que -y esto es quizá más grave- educan a una pequeña porción de los abogados colombianos. En el período 2001-2013, por ejemplo, solamente el $23 \%$ de los graduados vino de programas con doble rasero: $17 \%$ del sector privado y 6\% del oficial (MEN-SNIES, 2016).

En cambio, la mayoría de graduados viene de los programas ofrecidos por IES no acreditadas, sobre todo de los que no tienen el doble rasero. En el período 2001-2013, el 74\% de los graduados vino de instituciones no acreditadas, en general, y el $51 \%$ de pregrados sin el doble rasero, en particular.

\subsection{Los tipos de educación jurídica según calidad y costo}

Hasta ahora, se ha mostrado que existe una disparidad en términos de calidad de los pregrados. Dentro de esa disparidad, hay muy pocos

Es probable que estos 40 programas, según las tendencias mostradas aquí, también tengan un bajo desempeño en las pruebas Saber Pro. 
programas de alta calidad y en ellos estudia una porción pequeña de los abogados del país.

Al mismo tiempo, hay una gran disparidad en términos del costo de los pregrados. Así, se encontró que tanto en el sector privado como en el oficial predominan las matrículas con un costo por debajo del promedio. En el sector privado, por un lado, priman los pregrados de costo medio bajo $(64 \%)$, seguidos por los pregrados de costo medio alto (20\%). Muy pocos pregrados tienen un costo bajo $(4 \%)$, alto $(6 \%)$ o muy alto (5\%). Por otro lado, en el sector oficial, el $78 \%$ de los pregrados cobran matrículas de costo bajo y el $17 \%$ de costo medio bajo. ${ }^{12}$

Ahora bien, al cruzar las variables de calidad y costo, se obtienen cuatro niveles o tipos de pregrados de Derecho, en los que hay menores o mayores dificultades académicas de acceso. En estos niveles, la etiqueta de 'alta calidad' comprende solamente los pregrados con doble rasero de calidad y la de 'baja calidad' abarca a todos los demás, mientras que la etiqueta de 'alto costo' comprende las matrículas de costo medio alto, alto y muy alto, y la de 'bajo costo' abarca a las demás. Las principales características de estos niveles se observan en la tabla 1.

\section{Tabla 1. Caracterización económica y académica de los tipos} de pregrado en Derecho

\begin{tabular}{|c|c|c|c|}
\hline & \multicolumn{2}{|r|}{ Costo } \\
\hline & & Alto & Bajo \\
\hline \multirow{2}{*}{ 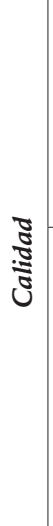 } & $\stackrel{\Xi}{\frac{D}{k}}$ & $\begin{array}{l}\text { Nivel A. Alta calidad y alto costo } \\
\text { - } 15 \text { pregrados ( } 8 \% \text { de la oferta) y } \\
12 \% \text { de los matriculados } \\
\text { - Selección económica alta } \\
\text { - Selección académica baja } \\
\text { Ejemplo: Eafit }\end{array}$ & $\begin{array}{l}\text { Nivel B. Alta calidad y bajo costo } \\
\text { - } 5 \text { pregrados ( } 3 \% \text { de la oferta) y } 4 \% \\
\text { de los matriculados } \\
\text { - Selección económica baja } \\
\text { - Selección académica muy alta } \\
\text { Ejemplo: Universidad Nacional de Colombia }\end{array}$ \\
\hline & 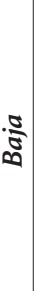 & $\begin{array}{l}\text { Nivel C. Baja calidad y alto costo } \\
\text { - } 30 \text { pregrados ( } 17 \% \text { de la oferta) } \\
\text { y } 14 \% \text { de los matriculados } \\
\text { - Selección económica medio alta } \\
\text { - Selección académica baja } \\
\text { Ejemplo: Universidad Católica de } \\
\text { Colombia }\end{array}$ & $\begin{array}{l}\text { Nivel D. Baja calidad y bajo costo } \\
\text { - } 128 \text { pregrados ( } 72 \% \text { de la oferta) y } \\
71 \% \text { de los matriculados } \\
\text { - Selección económica baja } \\
\text { - Selección académica muy baja en el } \\
\text { sector privado y media en el oficial } \\
\text { Ejemplo: Universidad Cooperativa de Co- } \\
\text { lombia (Bucaramanga) }\end{array}$ \\
\hline
\end{tabular}

Fuente: elaboración de la autora a partir de MEN-SNIES (2015).

12 No fue posible obtener información sobre el costo de matrícula de un pregrado privado y dos pregrados oficiales. 
Nivel A. Pregrados de alta calidad y alto costo. Este nivel acoge al $12 \%$ de los estudiantes matriculados y abarca 15 programas (es decir, el $8 \%$ del total de pregrados), todos ellos del sector privado. Hay una alta selección económica, pues 14 de los programas de este nivel tienen un costo alto o muy alto. De hecho, este es el nivel con el estrato socioeconómico promedio más alto de todos (estrato 4). Como se mostrará luego, los casos de estudiantes con baja capacidad económica que ingresan a este nivel son excepcionales.

Por otro lado, los programas de este nivel en general no tienen filtros académicos de ingreso particularmente selectivos o difíciles de superar. Esa selectividad puede ser medida a través de la tasa de absorción, que es la tasa que compara la cantidad de aspirantes con la de estudiantes finalmente admitidos. En este nivel, la tasa de absorción promedio entre los años 2007 y 2013 fue del 76\%.13

Nivel B. Pregrados de alta calidad y bajo costo. Este nivel abarca 5 programas oficiales y recibe al $4 \%$ de los estudiantes, lo que lo convierte en el nivel más pequeño de todos. En el acceso a este nivel hay poca selección económica, cosa que se revela en que los estudiantes pertenecen en promedio al estrato 2 y suelen pagar matrículas de costo bajo. Los estudiantes de estratos altos que ingresan a este nivel son ciertamente excepcionales. ${ }^{14}$

Al mismo tiempo, estos pregrados tienen una tasa de absorción promedio de apenas el 14\%, lo que significa que son altamente selectivos escolarmente. Los estudiantes que consiguen ser admitidos son los que tienen un mejor desempeño académico en los filtros de ingreso, y eso implica que cuentan con un mayor capital cultural dentro del total de aspirantes. Ese capital cultural está en muchos casos atado a la posesión de un cierto capital económico; por ejemplo, por la capacidad de costearse mejores colegios o cursos preuniversitarios.

Nivel C. Pregrados de baja calidad y alto costo. Este nivel comprende 30 programas privados (o sea, el $17 \%$ del total de pregrados) y

13 La información sobre la cantidad de aspirantes y admitidos fue obtenida del SNIES (2016), que solamente tenía datos disponibles sobre el período 2007-2013.

14 Por ejemplo, en el semestre 2015-1 en la Universidad Nacional, solamente el 1,5\% y el $0,1 \%$ de los estudiantes de Derecho pertenecían a los estratos 5 y 6, respectivamente (Universidad Nacional de Colombia, 2017). 
abarca al $14 \%$ de los estudiantes matriculados. Se trata de un nivel poco selectivo en términos académicos y medianamente selectivo en términos económicos: por un lado, los programas de este tipo admiten a la mayor parte de los aspirantes (con una tasa de absorción promedio del 82\%). Por otro lado, su estudiantado pertenece en promedio al estrato 3 y sus costos de matrícula están ligeramente por encima del promedio.

A este nivel están llamados a acceder los aspirantes que quizá no tienen grandes restricciones económicas, pero que carecen de los capitales (económico y cultural) para ingresar a los pregrados de alta calidad. Por lo demás, puede haber casos de estudiantes que sí tienen un alto capital económico, pero que deciden ingresar a este nivel (y no al nivel A), porque viven en las regiones y no desean trasladarse a una ciudad capital, por ejemplo.

Nivel D. Pregrados de baja calidad y bajo costo. En este nivel se concentra el grueso de la oferta y la demanda de educación jurídica, pues allí estudia el $71 \%$ de los estudiantes de Derecho y se ofrece el $72 \%$ de los programas. Así, en este nivel se ofrecen 128 pregrados: 31 oficiales y 97 privados.

Las matrículas de este nivel son todas de costo bajo y medio bajo, y sus estudiantes pertenecen en promedio al estrato 2. Por eso, puede decirse que hay poca selección desde el punto de vista económico. Por su parte, el grado de selección escolar varía según el sector, ya que en el sector oficial se admite en promedio al $47 \%$ de los aspirantes, mientras que en el sector privado se admite al $88 \%$ (de hecho, en muchos pregrados privados se admite al 100\% de los aspirantes). Este es, en principio, el nivel que tienen a su disposición aquellos que quedan excluidos de los demás niveles.

\section{El acceso a la educación jurídica de calidad y las desigualdades sociales}

La sección anterior mostró que la educación jurídica está segmentada en varios niveles o tipos de pregrados en Derecho. Esos niveles se distinguen por su costo, su calidad y sus condiciones de ingreso, y en ellos participan estudiantes con distintas estructuras de capital. 
Desde luego, ese esquema de segmentación o división por niveles recoge unas tendencias muy gruesas sobre los pregrados en Derecho en Colombia, entonces necesariamente deja por fuera algunos matices o casos intermedios..$^{15}$ A pesar de esas limitaciones, esa división es útil en la medida en que permite identificar las condiciones de acceso (o de exclusión) a cada tipo de pregrado. Con ese insumo, en esta sección se estudiarán con más detalle las características de los pregrados de mayor calidad (tabla 2), primero en el sector privado - nivel A- y luego en el sector oficial - nivel B-, para así mostrar cómo las oportunidades de acceder a una educación jurídica de calidad son muy limitadas y, como si fuera poco, están fuertemente atadas a las condiciones socioeconómicas de los estudiantes.

Tabla 2. Pregrados de alta calidad en el sector privado (nivel A) y en el sector público (nivel B)

\begin{tabular}{|l|l|l|c|l|}
\hline \multicolumn{1}{|c|}{ IES } & \multicolumn{1}{|c|}{ Seccional } & $\begin{array}{c}\text { Tasa de absorción } \\
\text { (2007-2013) }\end{array}$ & $\begin{array}{l}\text { Escala de } \\
\text { costo (2015) }\end{array}$ \\
\hline & Universidad Autónoma de Bucaramanga & Bucaramanga & $99 \%$ & Media alta \\
\hline Universidad de Bogotá Jorge Tadeo Lozano & Bogotá & $97 \%$ & Alta \\
\hline Universidad Icesi & Cali & $96 \%$ & Alta \\
\hline Universidad del Norte & Barranquilla & $93 \%$ & Alta \\
\hline Universidad de Medellín & Medellín & $92 \%$ & Alta ${ }^{16}$ \\
\hline \multirow{2}{*}{} & Universidad Santo Tomás & Bogotá & $90 \%$ & Alta \\
\hline Universidad Externado de Colombia & Bogotá & $89 \%$ & Muy alta \\
\hline Universidad Eafit & Medellín & $85 \%$ & Muy alta \\
\hline Universidad Pontificia Bolivariana & Medellín & $84 \%$ & Alta \\
\hline Pontificia Universidad Javeriana & Cali & $84 \%$ & Alta \\
\hline Universidad de La Sabana & Chía & $68 \%$ & Muy alta \\
\hline Universidad Sergio Arboleda & Bogotá & $46 \%$ & Muy alta \\
\hline
\end{tabular}

15 Este análisis deja por fuera, por ejemplo, los pregrados de costo y calidad intermedias, o las transiciones que los estudiantes hacen entre uno y otro tipo de pregrado según sus estrategias particulares de movilidad social.

16 Este programa tiene costos adecuables según el perfil socioeconómico de los estudiantes, pero, incluso frente a estudiantes con capacidades económicas medias, sus costos tienden a ubicarse en la escala alta. 


\begin{tabular}{|c|c|c|c|c|}
\hline & IES & Seccional & $\begin{array}{l}\text { Tasa de absorción } \\
\text { (2007-2013) }\end{array}$ & $\begin{array}{l}\text { Escala de } \\
\text { costo (2015) }\end{array}$ \\
\hline \multirow{3}{*}{$\begin{array}{l}\frac{\pi}{g} \\
\stackrel{z}{z}\end{array}$} & Universidad de los Andes & Bogotá & $41 \%$ & Muy alta \\
\hline & $\begin{array}{l}\text { Colegio Mayor Nuestra Señora del } \\
\text { Rosario }\end{array}$ & Bogotá & $41 \%$ & Muy alta \\
\hline & Pontificia Universidad Javeriana & Bogotá & $39 \%$ & Muy alta \\
\hline \multirow{5}{*}{$\begin{array}{l}\infty \\
\stackrel{0}{z} \\
z\end{array}$} & $\begin{array}{l}\text { Universidad Pedagógica y Tecnológica } \\
\text { de Colombia }\end{array}$ & Tunja & $25 \%$ & Baja \\
\hline & Universidad del Cauca & Popayán & $20 \%$ & Baja \\
\hline & Universidad de Cartagena & Cartagena & $14 \%$ & Baja \\
\hline & Universidad de Antioquia & Medellín & $9 \%$ & Baja \\
\hline & Universidad Nacional de Colombia & Bogotá & $4 \%$ & Baja \\
\hline
\end{tabular}

Fuente: elaboración de la autora a partir de MEN-SNIES (2015).

\subsection{La calidad cuesta (nivel A)}

Los pregrados de mayor calidad en el sector privado suelen a) tener débiles filtros académicos de ingreso, b) cobrar altos costos de matrícula, c) obtener mejores resultados en las pruebas Saber Pro mientras más costosas son sus matrículas, d) tener estudiantes de estratos medio altos o altos y e) estar concentrados territorialmente.

En primer lugar, la dificultad de los filtros académicos de este nivel tiende a ser baja, con una tasa de absorción promedio del $76 \% .{ }^{17} \mathrm{Co}^{-}$ mo muestra la tabla 2 , al menos 10 de los 15 pregrados de este nivel tuvieron una tasa de absorción superior al 80\%. Sin embargo, en otros 4 programas (todos de costo muy alto) la tasa de absorción fue menor al $50 \%$, de manera que en estos casos hay al mismo tiempo una selección económica alta y una selección académica media.

En segundo lugar, los pregrados de este nivel suelen tener matrículas costosas: 7 de ellos cobraban matrículas de costo alto y otros 7 de costo muy alto. Más aún, estos programas no solamente son costosos, sino que lo son cada vez más. Así, al menos 9 de los 15 pregrados de este nivel

17 La mayoría de programas privados establece requisitos de admisión adicionales al pago de la matrícula, como el buen desempeño en las pruebas Saber 11, en los exámenes de admisión institucionales o en las notas del bachillerato. 
aumentaron sus matrículas en más del $60 \%$ entre los años 2007 y 2014; y son, por mucho, los programas que más aumentaron sus matrículas en ese período (Observatorio de la Universidad Colombiana, 2014).

En tercer lugar, los pregrados de este nivel tienden a tener mejores promedios en las pruebas Saber Pro mientras más altas son sus matrículas. Como se explicó antes, los programas con doble rasero suelen tener los desempeños más destacados en estas pruebas; pero, dentro del sector privado, son los programas con doble rasero más costosos los que obtienen los puntajes más sobresalientes. Y, al contrario, hay una tendencia a que sea menor el promedio cuanto menor sea el costo de matrícula. Estos datos, unidos a la información sobre las acreditaciones, muestran que, con pocas excepciones, solo quien paga matrículas altas recibirá una educación privada de alta calidad.

En cuarto lugar, el estrato promedio de los pregrados de este nivel es el más alto de todos los niveles (estrato 4) y es el único nivel en el que hay pregrados estrato 5 . De hecho, estos programas no solamente tienen estratos más altos que los demás tipos de programas, sino que al menos 7 de ellos aumentaron su estrato promedio en el período 2010-2015 (MEN-SPADIES, 2016). Hay que decir que esto contrasta con la mayoría de programas ofrecidos por IES no acreditadas, que en promedio pertenecen a los estratos 2 y 3 . Parece haber, por lo tanto, una relación significativa entre la calidad de la educación y el alto costo; $\mathrm{y}$, tendencialmente, el estrato al que se pertenece.

La última característica es de orden territorial. En 2015, los pregrados privados en general se ofrecían en 25 departamentos y 40 municipios. Sin embargo, los pregrados del nivel A se ofrecían en solo seis ciudades y departamentos, y estaban particularmente concentrados en Bogotá (47\%), Medellín (20\%) y Cali (13\%). Así, para recibir una formación jurídica de alta calidad, es necesario tener la capacidad de pagar no solo las altas matrículas, sino también el costo de vida de las grandes ciudades.

Estas cinco características permiten concluir que los pregrados privados de alta calidad tienden a ser poco selectivos en términos académicos y muy selectivos desde el punto de vista económico. Eso supone que los aspirantes con mayor capacidad económica tienen amplias posibilidades de acceder a una buena formación en Derecho. Considerando la limitada oferta del sector oficial, quienes no tienen tal privilegio deben 
en cambio optar por el resto de la oferta privada de menor costo, pero también de menor calidad.

Ahora bien, es cierto que a estos pregrados del nivel A en ocasiones llegan estudiantes con créditos o becas, que no tienen una alta capacidad económica. Pero los casos de esos estudiantes tienden a ser excepcionales y se enfrentan con varias limitaciones. De un lado, los créditos suelen ser muy gravosos a futuro para los estudiantes y muchas veces establecen prerrequisitos difíciles de cumplir, como contar con un deudor solidario con historial crediticio favorable o tener un músculo financiero suficiente para amortizar las cuotas en el transcurso del pregrado.

De otro lado, las becas (como las de Ser Pilo Paga, que en realidad son créditos condonables) suelen tener un alcance muy limitado, al menos por dos razones: primero, porque benefician a muy pocos estudiantes; ${ }^{18}$ $\mathrm{y}$, segundo, porque se otorgan solo a los estudiantes con resultados académicos sobresalientes. Estas dos condiciones hacen que sean inalcanzables para los más excluidos. ${ }^{19}$ Así pues, estas opciones excepcionales no son suficientes para alterar la composición socioeconómica general de los pregrados de alto costo; y, sobre todo, no implican soluciones estructurales a los problemas de desigualdad educativa en Colombia.

El actual sistema de la educación jurídica, particularmente en el sector privado, es entonces un potente guardián de las jerarquías sociales existentes, pues premia las posiciones de origen de los estudiantes de élite. Esto implica que, tal como ocurre en la educación básica y media, la educación jurídica reproduce los privilegios de unos (de quienes estaban de antemano privilegiados socialmente), mientras que reproduce $\mathrm{o}$, incluso, profundiza las desigualdades sociales de otros (Bourdieu y Passeron, 1996, 2009).

18 Por ejemplo, en el 2015-I, el pregrado en Derecho de la Universidad Javeriana (Bogotá) recibió apenas 18 'pilos', entre un total de 1549 matriculados (MEN-SNIES, 2016; Pontificia Universidad Javeriana, 2016, p. 91); esto a pesar de que, según Correa y Pardo (2017), esa universidad es una de las que más estudiantes becados recibe en todo el país por cuenta de ese programa. El mejor de los casos, por mucho, es el de la Universidad Icesi, donde los 'pilos' alcanzan a ser una quinta parte del total de matriculados (Universidad Icesi, 2017).

19 Esto sin contar los otros muchos reparos que ha merecido el programa Ser Pilo Paga, porque, según algunos, contribuye a la privatización de la educación superior, al mismo tiempo que profundiza el desfinanciamiento de la universidad pública (Asmar Amador y Gómez Campo, 2015). 
Así, quienes tienen más dinero gozan de amplísimas oportunidades de acceder a una educación de mayor calidad y de obtener títulos académicos que probablemente estarán mejor cotizados en el mercado laboral. En este sentido, el éxito educativo y profesional de un estudiante no está determinado tanto por su esfuerzo como por su capital económico, factor que suele depender de 'accidentes de cuna' y con mucha frecuencia se escapa del control individual. Esa fuerte relación entre el capital económico de las personas y el tipo de educación que reciben lleva entonces a que se creen círculos viciosos de exclusión de los que tienen menos y círculos virtuosos de movilidad social para quienes gozan de altos ingresos económicos (Quintero, 2013, p. 19).

Este resultado es esperable, ya que el mercado tiende a replicar las desigualdades económicas que hay en la sociedad, y en este caso lo hace al condicionar la calidad de la educación a la capacidad de pago. Y si bien la posibilidad de 'comprar' educación de alta calidad no es en principio un problema, sí lo es cuando no se ofrecen oportunidades reales y suficientes para quienes no tienen con qué comprarla, como se mostrará a continuación.

\subsection{Lo público es insuficiente (nivel B)}

La estratificación de la educación jurídica privada quizá sería menos grave si, paralelamente, hubiera más educación pública de alta calidad y esta fuera ampliamente accesible. Pero en Colombia existe casi lo contrario, pues los pregrados oficiales de mayor calidad suelen a) tener filtros académicos de ingreso altamente selectivos, (b) cobrar bajos costos de matrícula, c) tener estudiantes de estratos bajos y d) estar concentrados territorialmente (tabla 2).

En primer lugar, los pregrados de este nivel tienen una disponibilidad de cupos muy reducida y, por eso, establecen filtros académicos de ingreso que muy pocos estudiantes superan (el 14\%, en promedio). ${ }^{20}$ En el período 2007-2013, por ejemplo, el pregrado de Derecho de la

20 El filtro puede consistir en la obtención de altos puntajes bien en las pruebas Saber 11 (como en la UPTC), bien en el examen de admisión institucional (como en la Universidad Nacional o de Cartagena). 
Universidad de Antioquia (Medellín) admitió al 9\% de los aspirantes, mientras que la Universidad Nacional admitió apenas al $4 \%$.

En segundo lugar, en los pregrados de este nivel (como en buena parte de los pregrados oficiales) no hay una matrícula preestablecida, sino que el costo varía según el perfil socioeconómico de cada estudiante. Se buscó entonces el promedio de matrícula pagado por los estudiantes en 2015 en los pregrados oficiales de alta calidad y en todos ellos se halló que los estudiantes pagaron en promedio matrículas de costo bajo (de hecho, pagaron menos de un salario mínimo legal mensual).

En tercer lugar, se encontró que 4 de los 5 pregrados que componen este nivel pertenecían en promedio al estrato 2 y solamente uno (el pregrado de la Universidad Nacional) era estrato 3. Este dato, unido a los anteriores, permite afirmar que en los programas oficiales de este nivel hay una alta selección académica, pero una baja selección económica.

Por último, hay que decir que, si bien la educación jurídica oficial ha conseguido una relativa desconcentración territorial (en 2015, cubría 20 departamentos y 32 municipios), los pregrados oficiales de alta calidad se concentran en apenas cinco ciudades capitales. Esto, por las razones advertidas antes, supone límites económicos que pueden ser desproporcionados para los aspirantes de las regiones.

Estas características del sector oficial revelan que, aunque de forma menos evidente que en el sector privado, en la educación pública se presenta también una cierta reproducción de las desigualdades sociales. El filtro académico de ingreso no está medido por la alta capacidad de pago, sino por las capacidades escolares del estudiante. La justificación que tradicionalmente se le ha atribuido a este tipo de filtro es que permite que los estudiantes ingresen gracias a su mérito. Según esto, los exámenes estandarizados ( $\mathrm{y}$, en general, las pruebas académicas) son medidores objetivos y neutrales, que buscan premiar la excelencia escolar de los estudiantes sin distingo de su origen social. De esa manera se igualan las posibilidades de ingreso de todos los aspirantes.

Sin embargo, cada vez son más los estudios que, en línea con la teoría de la reproducción social, muestran que los medidores del mérito distan de ser neutrales. Su ropaje de igualdad formal nos hace creer que se están brindando a todos los aspirantes las mismas oportunidades y que la selección no se funda en calidades estatutarias o de nacimiento. Pero esa 
idea meritocrática de la movilidad social no es otra cosa que una ilusión que hace que las distinciones (sociales y culturales) sean vistas como 'naturales', y es así como se encubren los privilegios que están por debajo de esa ilusión de igualdad (Bourdieu y Passeron, 2009, p. 104).

En el caso colombiano, dichos estudios revelan, por ejemplo, que hay una correlación entre el origen social de los estudiantes y su desempeño educativo en los niveles básico y medio. ${ }^{21}$ Los estudiantes que tienen mayor chance de obtener un alto desempeño académico son los egresados de los colegios privados costosos. Estos egresados no solamente reciben una mejor formación en los valores y aptitudes que evalúa el sistema educativo dentro de sus colegios, sino que suelen recibir mayores capitales sociales y culturales de su ambiente familiar; por ejemplo, por el hecho de que sus padres sean profesionales y dispongan de tiempo para reforzar dentro del hogar el aprendizaje de sus hijos. En cambio, los estudiantes que están peor ubicados en la jerarquía socioeconómica suelen recibir un menor capital cultural durante su educación media y, por eso, obtienen resultados más pobres en sus notas escolares y en las pruebas estatales (Sánchez y Otero, 2012). Existe, por lo tanto, una fuerte correlación entre el desempeño educativo y el origen social de los estudiantes.

En este orden de ideas, mientras más alto sea el origen socioeconómico de un estudiante, mejor equipado estará para enfrentar los esquemas de selección de la educación superior. Así, los estudiantes que logran superar las difíciles pruebas de admisión de la universidad pública son probablemente quienes, entre los demás aspirantes, gozan de un mayor capital cultural y económico. Es decir, quienes pudieron costearse un mejor colegio, quienes recibieron mayores capitales de su familia (la biblioteca y los computadores disponibles en el hogar, el lenguaje y los repertorios culturales de los padres, etc.), quienes tuvieron la posibilidad (económica, familiar, etc.) de presentar varias veces el examen de admisión; e, incluso, quienes pudieron costearse un curso preuniversitario.

21 Ver, entre otros, Barrera Osorio, Maldonado y Rodríguez (2012); Gamboa y Waltenberg (2011); García Villegas, Espinosa, Jiménez Ángel y Parra Heredia (2013); Gaviria (2002); López Mera (2010); Sánchez y Otero (2012). 
Se trata, nuevamente, de una reproducción de los privilegios de unos y de las desigualdades de otros.

\section{3. 'La elección de los elegidos'}

Las oportunidades de acceder a una educación jurídica de calidad están fuertemente marcadas por el perfil socioeconómico de los aspirantes, de forma directa en el caso de la educación privada de calidad e indirecta en la pública de calidad. De una parte, las personas con capital económico alto o medio alto tienen la posibilidad y la capacidad de explorar todas las opciones o tipos de pregrado, y entre esas opciones está la oferta de mayor calidad. Por ejemplo, suponiendo que no superara las pruebas de admisión en los programas oficiales de calidad, un estudiante de clase alta tendrá la opción de costearse un programa privado de alta calidad. Como se sabe, los filtros académicos de la oferta privada no son muy estrictos, así que serán pocos los aspirantes con alto capital económico que no podrán acceder a una educación jurídica de alta calidad.

En contraste, las personas con bajo capital económico ven mucho más limitadas sus oportunidades de acceder a una educación jurídica de alta calidad. Las posibilidades de obtener recursos para acceder al nivel A son pocas y suelen ser gravosas, mientras que la probabilidad de ingresar al nivel B es bajísima. Sus mayores opciones están entonces concentradas en el nivel $C y$, para la mayoría, en el nivel $D$.

Al plantear el panorama del acceso a la educación jurídica desde el punto de vista de la igualdad de oportunidades, se pone nuevamente de presente cómo el medio social en el que una persona nace, crece y adquiere unos ciertos capitales (pre)determina poderosamente su destino educativo y profesional como abogado. El sistema actual de educación jurídica reproduce las desigualdades sociales, pues privilegia los valores, calidades o capacidades de quienes gozan de una mayor herencia económica y cultural.

Eso es grave porque no solo supone en sí mismo una reproducción de las desigualdades de origen, sino que, como se anunció antes, es una reproducción con vocación de extenderse en el tiempo. Los estudiantes que reciben una mejor formación jurídica podrán tener mayor éxito académico y ostentarán títulos con un alto capital simbólico, y eso posi- 
blemente les permitirá contar con mejores oportunidades profesionales y recibir una mejor remuneración que el resto de estudiantes que no tuvieron iguales oportunidades. Parafraseando el diagnóstico de Bourdieu y Passeron (2009, pp. 12 y ss.) sobre el sistema educativo francés, en la educación jurídica de calidad se refuerza la elección de los elegidos; y, al contrario, se profundiza la exclusión de los que ya estaban excluidos.

\section{Conclusiones}

Los resultados presentados aquí se pueden resumir en dos grandes conclusiones. La primera es que la oferta de educación jurídica es muy amplia y dispar, de manera que hoy se ofrecen pregrados casi de todas las calidades y costos posibles, aunque hay un claro predominio de los pregrados de bajo costo y baja calidad. Paralelamente, hay una creciente demanda de educación jurídica. Los estudiantes se gradúan entonces con los perfiles más disímiles, aunque predominan los estudiantes de menor calidad.

La segunda conclusión es que el medio social de origen y los capitales (principalmente el económico) de un estudiante predeterminan poderosamente sus posibilidades de recibir una buena educación jurídica; y ello, a su turno, es determinante para definir su trayectoria profesional. Así pues, a los pregrados de alta calidad, con pocas excepciones, solo acceden quienes tienen alta capacidad de pago (en el sector privado) o resultados académicos sobresalientes (en el sector público). Esto sugiere que la educación jurídica, en lugar de reducir las desigualdades sociales, las reproduce.

De acuerdo con lo anterior, una de las muchas preguntas que quedan por resolver es si esta división entre los distintos tipos de educación jurídica se reproduce o se refleja en las trayectorias profesionales de los abogados. Por ejemplo, ¿los bufetes se ocupan de asuntos y clientes diferentes según el perfil socioeconómico de sus abogados o sus universidades de procedencia? $\mathrm{O}$, incluso, ¿los abogados ocupan distintos cargos y perciben distintos salarios según su universidad de pregrado? ¿Cuáles son los egresados que predominan en las entidades del Estado? Siguiendo a 
Bourdieu (2013), ¿acaso se está formando una pequeña y gran nobleza de Estado en los distintos tipos de programas?

Pero, incluso si no incidiera en el éxito profesional de los abogados, la mera provisión desigual de la educación de calidad supone una franca violación del derecho a la educación y del derecho a la igualdad de oportunidades, pues, en un sistema educativo equitativo, el origen social no debería determinar (al menos no de manera tan definitiva) la posibilidad de acceder a una educación de buena calidad. La educación superior no debería servir a la reproducción o preservación de las jerarquías sociales, sino que debería ser un espacio para la emancipación; la plataforma por excelencia de la movilidad social. Un espacio que permita combatir las diferencias y democratizar las oportunidades para los grupos sociales menos privilegiados (Arango Gaviria, 2006; Chan de Ávila, García Peter y Zapata Galindo, 2013).

Ahora bien, este caso de la educación jurídica se enmarca en un contexto más amplio de desregulación y autonomía de la educación superior en general (Ley 30 de 1992), que le dio mayor margen de acción al mercado y redujo el papel del Estado. Dentro de ese esquema de desregulación generalizada, se han profundizado las brechas de calidad entre los programas más costosos y los más baratos, sin que paralelamente se haya expandido la educación pública de calidad (Gómez Campo, 2000). El caso de los pregrados de Derecho encaja bien en esa descripción: la oferta de calidad del sector privado es costosa y la del sector oficial es insuficiente, mientras que priman la oferta y la demanda de baja calidad y bajo costo. Como se anunciaba en la introducción, esta situación sería en sí misma preocupante en el caso de cualquier carrera, pero es especialmente grave en el caso de los abogados, porque, una vez graduados, ellos serán jueces, procuradores, litigantes y burócratas, y tendrán en sus manos bienes tan importantes como la transparencia estatal, el acceso a la justicia de los ciudadanos o el derecho a la libertad de sus defendidos.

En vista de que el mercado no ha contribuido por sí mismo a aliviar esta situación, es preciso que el Estado ataque el problema desde varios frentes. En primer lugar, debe aumentar y descentralizar la oferta oficial de programas jurídicos de calidad y procurar que sus niveles de acreditación superen por mucho los del sector privado. Y, llegado el caso de que no tenga la capacidad de ampliar suficientemente la oferta oficial, deberá 
cuando menos promover programas integrales e incluyentes de subsidios a la demanda. Por último, debe regular y controlar que la totalidad de los programas privados cumpla aunque sea unos estándares medios de calidad. Es inadmisible que, siguiendo la lógica del mercado y a falta de controles estatales, la mayoría de programas ofrezcan educación jurídica a bajo costo, pero solamente porque están poco dispuestos a invertir en el fortalecimiento de esa educación.

Es necesario, sin embargo, que estos frentes sean atacados simultáneamente, para que no se repita lo que ha ocurrido con los más recientes intentos estatales de subir los estándares de calidad de la educación jurídica (Ministerio de Educación y Ministerio de Justicia y del Derecho, 2016). En estos casos, algunos representantes de los programas de baja calidad y bajo costo se han opuesto a esos proyectos, alegando que el endurecimiento de los estándares tiene tintes elitistas, pues necesariamente llevará a un encarecimiento de los costos de matrícula. Si la educación oficial es insuficiente y el grueso de la educación privada deja de ser barata -alegan ellos-, entonces se privaría a un sinnúmero de estudiantes de sectores populares de la posibilidad de estudiar Derecho y la abogacía volvería a ser una profesión reservada para las élites.

El Ministerio de Educación está, por lo tanto, en mora de expandir la educación oficial de calidad y de proponer mejoras a la calidad del sector privado sin aumentar sus costos, y es por eso que al Estado le cuesta tanto evitar que este tipo de cuestionamientos (que tienen mucho de cierto) bloqueen sus proyectos regulatorios. No obstante, podría pensarse también que ese bloqueo regulatorio es útil a los intereses del Estado, porque, en cierta medida, esa oferta de bajo costo oculta la incapacidad estatal de responder a la creciente demanda de educación jurídica. Invirtiendo poco en educación pública pero siendo connivente con la educación privada, el Estado consigue engrosar las cifras sobre acceso a la educación superior de los sectores populares, mientras invisibiliza los problemas de calidad (y probablemente de inmovilidad social) que vienen detrás.

Los proyectos que buscan aumentar los requisitos de calidad probablemente implicarían cerrar algunos programas o impedir la creación de programas nuevos, y es cierto que ello puede truncar las opciones de acceso de algunas personas que aspiran a ser abogadas. Sin embargo, esos proyectos no deberían ser condenados de antemano, pues hay que 
recordar que allí están en juego no solo el derecho a la educación de los estudiantes, sino también los muchos derechos de los usuarios de los futuros abogados. En un contexto como el colombiano, donde hay grandes desigualdades estructurales y donde los abogados cumplen un rol social tan fundamental, el gran reto del Estado (y, por qué no, de la academia) es entonces diseñar esquemas que aseguren la alta calidad de la educación jurídica sin que ello afecte desproporcionadamente a los que tienen menos.

\section{Referencias}

Arango Gaviria, L. G. (2006). Jóvenes en la universidad: género, clase e identidad profesional. Bogotá: Siglo del Hombre Editores-Universidad Nacional de Colombia.

Arbeláez Herrera, N. C., \& Espinosa Pérez, B. (2002). Retos en la formación de abogados. Propuestas desde la universidad. Criterio Jurídico, (2), 161-186.

Asmar Amador, M. P., \& Gómez Campo, V. M. (2015). Ser Pilo Paga: política de privatización y desfinanciamiento de la universidad pública. Recuperado de http://universidad.edu.co/index.php/historial-de-noticias/64-notici as-2015/12033-2015-10-22-10-27-37

Barrera Osorio, F., Maldonado, D., \& Rodríguez, C. (2012). Calidad de la educación básica y media en Colombia: diagnóstico y propuestas. Bogotá: Universidad de los Andes.

Boudon, R. (1983). La desigualdad de oportunidades. La movilidad social en las sociedades industriales. Barcelona: Laia.

Bourdieu, P. (2013). La nobleza de Estado. Educación de élite y espíritu de cuerpo. Buenos Aires: Siglo XXI Editores.

Bourdieu, P., \& Passeron, J.-C. (1996). La reproducción. Elementos para una teoría del sistema de enseñanza. Barcelona: Fontamara.

Bourdieu, P., E Passeron, J.-C. (2009). Los herederos. Los estudiantes y la cultura ( $2^{\text {a }}$ ed.). Buenos Aires: Siglo XXI Editores.

Chan de Ávila, J., García Peter, S., \& Zapata Galindo, M. (2013). Incluyendo sin excluir: género y movilidad en la educación superior. En J. Chan de Ávila, S. García Peter \& M. Zapata Galindo (Eds.), Incluyendo sin excluir. Género y movilidad en instituciones de educación superior (pp. 7-15). Berlín: Tranvia.

Colombia, Consejo de Estado. (2011). Sentencia 11001032400020070038600. 
Colombia, Ministerio de Educación y Ministerio de Justicia y del Derecho. (2016). Memoria administrativa y propuesta de resolución "mediante la cual se definen las características específicas de calidad de los programas de pregrado en Derecho".

Correa, P., E Pardo, T. (29 de abril de 2017). Los Andes, ¿la universidad pública más costosa del país? El Espectador. Recuperado de http://www.elespectador. com/noticias/educacion/los-andes-la-universidad-publica-mas-costosa-delpais-articulo-691584

DANE. (2005). Colombia. Estimaciones de población 1985-2005 y proyecciones de población 2005-2020. Recuperado de http://www.dane.gov.co/files/investigaciones/poblacion/proyepobla06_20/ProyeccionMunicipios2005_2020.xls DNP. (2014). Bases del Plan Nacional de Desarrollo 2014-2018. Recuperado de https://colaboracion.dnp.gov.co/CDT/Prensa/PND\%202014-2018\%20 Bases\%20Final.pdf

Espinosa Blanco, Y. A. (2012). Masificación y control de la educación legal en Colombia y Argentina: una perspectiva comparada (Tesis de maestría, Universidad Nacional de Colombia, Bogotá).

European Commission. (2016). The 2016 EU Justice Scoreboard. Luxemburgo: European Union. Recuperado de http://ec.europa.eu/justice/effective-justice/ files/justice_scoreboard_2016_en.pdf

Fuentes Hernández, A. (2005). Educación legal y educación superior en Colombia: desarrollos institucionales y legales 1990-2002. Sistemas Judiciales -CELA-Inecip, (9), 39-51.

Gamboa, L. F., E Waltenberg, F. (2011). Inequality of opportunity in educational achievement in Latin America: evidence from PISA 2006-2009. Bogotá: Universidad del Rosario, Facultad de Economía.

García Villegas, M. (2010). Sociología de la profesión jurídica. En Los abogados en Colombia (pp. 9-25). Bogotá: Universidad Nacional de Colombia, Facultad de Derecho, Ciencias Políticas y Sociales.

García Villegas, M., Espinosa, J. R., Jiménez Ángel, F., E Parra Heredia, J. D. (2013). Separados y desiguales. Educación y clases sociales en Colombia. Bogotá: Dejusticia. Gaviria, A. (2002). Los que suben y los que bajan. Educación y movilidad social en Colombia. Bogotá: Fedesarrollo-Alfaomega.

Giddens, A. (1997). Sociología. Madrid: Alianza Editorial.

Gómez Campo, V. M. (2000). Cuatro temas críticos de la educación superior en Colombia. Bogotá: Alfaomega-Ascun-Universidad Nacional de Colombia.

Gómez Campo, V. M. (19 de noviembre de 2014). ¿Quién vigila a las universidades? Las 2 orillas. Recuperado de http://www.las2orillas.co/quien-vigilalas-universidades/ 
Goux, D., E Maurin, É. (1997). Destinées sociales: le role de l'Ecole et du milieu d'origine. Economie et Statistique, 306(1), 13-26.

López Mera, S. (2010). El efecto colegio en Colombia: tres décadas de estudio. Equidad y Desarrollo, 14, 85-101.

MEN-SNIES. (2015). Búsqueda de programas de instituciones de educación superior.

Recuperado de http://snies.mineducacion.gov.co/consultasnies/programa MEN-SNIES. (2016). Estadística - Consultas avanzadas.

MEN-SNIES. (2016). Sistema Nacional de Información de la Educación Superior. Recuperado de http://www.mineducacion.gov.co/sistemasdeinformacion/1735/ w3-propertyname-2672.html

MEN-SPADIES. (2016). Caracterización estudiantil.

Observatorio de la Universidad Colombiana. (2014). Lo que deben pagar los primíparos en programas de Derecho. Recuperado de http://www.universidad.edu. co/index.php?option=com_contentyview=articleyid=5229:lo-que-debenpagar-los-primiparos-en-programas-de-derechoycatid=16:noticiasyltemid=198 Observatorio de la Universidad Colombiana. (2015). ¿Está desacreditada la acreditación de alta calidad? Recuperado de http://www.universidad.edu. co/index.php?option=com_contentyview=articleyid=6260:2015-10-19-1200-11 ycatid $=16$ : noticiasyltemid $=198$

Pérez Perdomo, R. (2005). Educación jurídica, abogados y globalización en América Latina. Sistemas Judiciales -CELA-Inecip, (9), 4-14.

Pontificia Universidad Javeriana. (2016). Informe del rector al Consejo de Regentes 2015. Bogotá: PUJ.

Quintero, Ó. A. (2013). Racisme et discrimination à l'université. Lectures croisées des societés française et colombienne à partir de l'experience vécue des étudiants noirs à Paris et Bogota (Tesis doctoral, Université Rennes 2, École Doctorale Sciences Humaines et Sociales, Rennes, Francia).

Salamanca, F. A. (2010a). Educación legal en Colombia. Análisis cuantitativo desde una perspectiva histórica. En Los abogados en Colombia (pp. 103-143). Bogotá: Universidad Nacional de Colombia, Facultad de Derecho, Ciencias Políticas y Sociales.

Salamanca, F. A. (2010b). Reseña histórica de la regulación de la enseñanza del Derecho. En M. García Villegas (Ed.), Los abogados en Colombia (pp. 61-88). Bogotá: Universidad Nacional de Colombia.

Sánchez, A., E Otero, A. (2012). Educación y reproducción de la desigualdad en Colombia. Reportes del Emisor. Investigación e Información Económica, (154), 1-4. Silva García, G. (2001). El mundo real de los abogados y de la justicia. Bogotá: Universidad Externado de Colombia. 
Unidad de Registro Nacional de Abogados. (2009). Gaceta del foro. Novedades del Registro Nacional de Abogados. Julio a noviembre de 2009. Bogotá: Unidad de Registro Nacional de Abogados-Consejo Superior de la Judicatura.

Unidad de Registro Nacional de Abogados. (2014). Respuesta a derecho de petición sobre tarjetas profesionales vigentes.

Universidad Icesi. (2017). Respuesta a derecho de petición.

Universidad Nacional de Colombia. (2017). Respuesta a derecho de petición. 